\title{
ON THE COEFFICIENTS OF BAZILEVIČ FUNCTIONS
}

\author{
F. R. KEOGH ${ }^{1}$ AND SANFORD S. MILLER
}

Aвstract. Let $B(\alpha)$ denote the class of normalized $(f(0)=0$, $\left.f^{\prime}(0)=1\right)$ Bazilevič functions of type $\alpha$ defined in $\Delta:|z|<1$, i.e. $f(z)$ $=\left[\alpha \int_{0}^{2} P(\zeta) g(\zeta)^{\alpha} \zeta^{-1} d \zeta\right]^{1 / \alpha}$ where $g(\zeta)$ is starlike in $\Delta, P(\zeta)$ is regular with $\operatorname{Re} P(\zeta)>0$ in $\Delta$ and $\alpha>0$. Let $B_{m}(\alpha)$ denote the subclass of $B(\alpha)$ which is $m$-fold symmetric $\left(f\left(e^{2 \pi i / m} z\right)=e^{2 \pi i / m} f(z), m=1,2, \cdots\right)$. Functions in $B(\alpha)$ have been shown to be univalent. The authors obtain sharp coefficient inequalities for functions in $B_{m}(1 / N)$ where $N$ is a positive integer. In addition an example of a Bazilevic function which is not close-to-convex is given.

Let $S$ denote the class of functions

$$
f(z)=z+\sum_{n=2}^{\infty} a_{n} z^{n}
$$

that are univalent in the unit disk $\Delta:|z|<1$. If $g(z)$ is starlike in $\Delta$, $P(z)$ is regular with $\operatorname{Re} P(z)>0$ in $\Delta$ and $\alpha>0$, then the function

$$
f(z)=\left[\alpha \int_{0}^{z} P(\zeta) g(\zeta)^{\alpha} \zeta^{-1} d \zeta\right]^{1 / \alpha}
$$

has been shown by Bazilevič [1] (see also Pommerenke [6]) to be a regular and univalent function in $\Delta$. The powers appearing in the formula are meant as principal values. We shall call a function in $S$ satisfying (1) a Bazilevič function of type $\alpha$ and denote the class of such functions by $B(\alpha)$. Note that $B(1)$ is the class of normalized close-to-convex functions.

A function $f(z)$ analytic in $\Delta$ is said to be $m$-fold symmetric ( $m$ $=2,3, \cdots)$ if $f\left(e^{2 \pi i / m} z\right)=e^{2 \pi i / m} f(z)$. In particular, every odd $f(z)$ is 2fold symmetric. Let $S_{m}$ denote the subclass of $S$ consisting of those $f(z)$ that are $m$-fold symmetric. We similarly define $B_{m}(\alpha)$. A simple argument shows that $f \in S_{m}$ is characterized by having a power series of the form $f(z)=z+a_{m+1} z^{m+1}+a_{2 m+1} z^{2 m+1}+\cdots$.

The Bieberbach conjecture remains unsettled for functions in $B(\alpha)$ except for the case $\alpha=1 / N$, where $N$ is a positive integer (Zamorski

Received by the editors September 8, 1970.

AMS 1970 subject classifications. Primary 30A32, 30A34.

Key words and phrases. Univalent, Bazilevi $x$ functions, close-to-convex functions, $m$-fold symmetric, Bieberbach conjecture, majorization.

1 The research of this author was supported by the National Science Foundation under grant number GP-19533. 
[7]). C. Pommerenke [5] has obtained sharp coefficient inequalities for functions in $B_{m}(1)$. In this paper we shall be concerned mainly with obtaining sharp coefficient inequalities for functions in $B_{m}(1 / N)$. The method is different from the methods of Zamorski and Pommerenke and our results include theirs.

Many of the properties of Bazilevič functions of type $\alpha, 0<\alpha<1$, coincide with properties of close-to-convex functions, including some of the results of this paper. We will show, at the end of the paper, an example of a Bazilevič function which is not close-to-convex.

The notation $g(z) \ll h(z)$ (" $g(z)$ is majorized by $f(z)$ ") will mean that if $g(z)=\sum_{0}^{\infty} b_{n} z^{n}$ and $h(z)=\sum_{0}^{\infty} c_{n} z^{n}$ then $\left|b_{n}\right| \leqq c_{n}$ for $n=0,1, \ldots$ (see, for example, [3, p. 69]).

We shall need the following lemmas, the first of which is well known.

Lemma 1. (a) $\phi \in S_{m}$ if and only if $\phi(z)=\left[f\left(z^{m}\right)\right]^{1 / m}$ where $f \in S$. (b) $\phi(z)$ is $m$-fold symmetric and starlike if and only if $\phi(z)=\left[f\left(z^{m}\right)\right]^{1 / m}$ where $f(z)$ is starlike.

LEMMA 2. If $f(z)$ is $m$-fold symmetric and starlike and $\gamma>0$ then

$$
[f(z) / z]^{\gamma} \ll\left|f^{\prime}(0)\right| \gamma\left(1-z^{m}\right)^{-2 \gamma / m} .
$$

Proof. A function $f(z)$ which is $m$-fold symmetric and starlike has a Herglotz representation given by

$$
\log \frac{f(z)}{z f^{\prime}(0)}=\int_{0}^{2 \pi} \log \frac{1}{\left(1-z^{m} e^{-i \phi}\right)^{2 / m}} d \mu(\phi),
$$

where $\mu(\phi)$ is nondecreasing on $[0,2 \pi]$ and $\mu(2 \pi)-\mu(0)=1$. Thus

$$
\begin{aligned}
\log \frac{f(z)}{z f^{\prime}(0)} & =\frac{2}{m} \int_{0}^{2 \pi} \sum_{n=1}^{\infty} \frac{z^{m n} e^{-i n \phi}}{n} d \mu(\phi) \\
& =\frac{2}{m} \sum_{n=1}^{\infty}\left[\int_{0}^{2 \pi} e^{-i n \phi} d \mu(\phi)\right] \frac{z^{m n}}{n} \ll \frac{2}{m} \sum_{n=1}^{\infty} \frac{z^{m n}}{n},
\end{aligned}
$$

and consequently

$$
\log \left(f(z) / z f^{\prime}(0)\right) \ll \log \left(1-z^{m}\right)^{-2 / m} .
$$

If $\gamma>0$, then

$$
\gamma \log \left(f(z) / z f^{\prime}(0)\right) \ll \gamma \log \left(1-z^{m}\right)^{-2 / m},
$$

or

$$
\log \left[f(z) / z f^{\prime}(0)\right]^{\gamma} \ll \log \left(1-z^{m}\right)^{-2 \gamma / m} .
$$


Since exponentiation preserves the majorization property we obtain $\left[f(z) / z f^{\prime}(0)\right]^{\gamma} \ll\left(1-z^{m}\right)^{-2 \gamma / m}$, which is equivalent to the desired result.

THEOREM 1. $\phi(z) \in B_{m}(\alpha)$ if and only if

$$
\phi(z)=\left[f\left(z^{m}\right)\right]^{1 / m}
$$

wheref $f(z) \in B_{1}(\alpha / m)$.

Proof. (i) If $\phi(z) \in B_{m}(\alpha)$, then on differentiating (1) we obtain

$$
z \phi^{\prime}(z) \phi(z)^{\alpha-1}=g(z)^{\alpha} P(z),
$$

where $g$ is starlike and $\operatorname{Re} P(z)>0$. By Lemma 1 (a) there exists an $f \in S$ satisfying (2), and substitution of (2) in (3) yields

$$
z^{m} f^{\prime}\left(z^{m}\right) f\left(z^{m}\right)^{\alpha / m-1}=g(z)^{\alpha} P(z) .
$$

Replacing $z$ by $e^{2 k \pi i / m} z$ we have

$$
z^{m} f^{\prime}\left(z^{m}\right) f\left(z^{m}\right)^{\alpha / m-1}=g\left(e^{2 k \pi i / m} z\right)^{\alpha} P\left(e^{2 k r i / m} z\right),
$$

for $k=0,1, \cdots, m-1$. If we multiply the $m$ equations in (4) and then take the $m$ th root we obtain

$$
z^{m} f^{\prime}\left(z^{m}\right) f\left(z^{m}\right)^{\alpha / m-1}=\left[\prod_{k=0}^{m-1} g\left(e^{2 k \pi i / m} z\right)\right]^{\alpha / m}\left[\prod_{k=0}^{m-1} P\left(e^{2 k r i / m} z\right)\right]^{1 / m} .
$$

It is easily verified that the function [[ $\left.\prod_{k=0}^{m-1} g\left(e^{2 k \pi i / m} z\right)\right]^{1 / m}$ is $m$-fold symmetric and starlike and hence, by Lemma 1 (b), can be written as $\left[h\left(z^{m}\right)\right]^{1 / m}$ where $h$ is starlike. It is also easily verified that

$$
\left[\prod_{k=0}^{m-1} P\left(e^{2 k \pi i / m z}\right)\right]^{1 / m}=c_{0}+c_{m} z^{m}+\cdots=Q\left(z^{m}\right),
$$

say, where $\operatorname{Re} Q(z)>0$. Thus we have

$$
z^{m} f^{\prime}\left(z^{m}\right) f\left(z^{m}\right)^{\alpha / m-1}=h\left(z^{m}\right)^{\alpha / m} Q\left(z^{m}\right), \quad z f^{\prime}(z) f(z)^{\alpha / m-1}=h(z)^{\alpha / m} Q(z),
$$

or $f \in B(\alpha / m)$.

(ii) Conversely, if $f \in B(\alpha / m)$ we have

$$
z f^{\prime}(z) f(z)^{\alpha / m-1}=h(z)^{\alpha / m} Q(z),
$$

where $h$ is starlike and $\operatorname{Re} Q(z)>0$. Thus

$$
z^{m} f^{\prime}\left(z^{m}\right) f\left(z^{m}\right)^{\alpha / m-1}=h\left(z^{m}\right)^{\alpha / m} Q\left(z^{m}\right)
$$

and, if we let $\phi(z)=\left[f\left(z^{m}\right)\right]^{1 / m}$, we obtain

$$
z \phi^{\prime}(z) \phi(z)^{\alpha-1}=\left[h\left(z^{m}\right)^{1 / m}\right]^{\alpha} Q\left(z^{m}\right) .
$$


By Lemma $1\left(\right.$ b),$g(z)=\left[h\left(z^{m}\right)\right]^{1 / m}$ is ( $m$-fold symmetric) starlike, and if we write $P(z)=Q\left(z^{m}\right)$ we have

$$
z \phi^{\prime}(z) \phi(z)^{\alpha-1}=g(z)^{\alpha} P(z) .
$$

Thus $\phi(z) \in B_{m}(\alpha)$, and this completes the proof of the theorem.

THEOREM 2. If $\phi(z) \in B_{m}(\alpha)$ then $[\phi(z) / z]^{\alpha} \ll\left(1-z^{m}\right)^{-2 \alpha / m}$.

Proof. The function

$$
F(z)=[\phi(z) / z]^{\alpha}=1+A_{1} z^{m}+A_{2} z^{2 m}+\cdots
$$

satisfies the differential equation

$$
z F^{\prime}(z)+\alpha F(z)=\alpha \phi^{\prime}(z) \phi(z)^{\alpha-1} / z^{\alpha-1},
$$

or, using (5),

$$
z F^{\prime}(z)+\alpha F(z)=\alpha\left\{\left[h\left(z^{m}\right)\right]^{1 / m} / z\right\}^{\alpha} Q\left(z^{m}\right),
$$

where $\left[h\left(z^{m}\right)\right]^{1 / m}$ is $m$-fold symmetric and starlike and $\operatorname{Re} Q\left(z^{m}\right)>0$. Hence, by Lemma 2, we have

$$
\left\{\left[h\left(z^{m}\right)\right]^{1 / m} / z\right\}^{\alpha} \ll\left|h^{\prime}(0)\right|^{\alpha / m}\left(1-z^{m}\right)^{-2 \alpha / m} .
$$

Also, if $Q\left(z^{m}\right)=c_{0}+c_{m} z^{m}+c_{2 m} z^{2 m}+\cdots$ then

$$
Q\left(z^{m}\right) \ll\left|c_{0}\right| \frac{1+z^{m}}{1-z^{m}}
$$

$[4$, p. 170]. Since multiplication preserves the majorization property, (6), (7) and (8) yield

$$
z F^{\prime}(z)+\alpha F(z) \ll \alpha\left|h^{\prime}(0)\right| \alpha / m\left|c_{0}\right| \frac{1+z^{m}}{\left(1-z^{m}\right)^{1+2 \alpha / m}} .
$$

Setting $z=0$ in (6) we obtain $\alpha=\alpha\left[h^{\prime}(0)\right]^{\alpha / m} c_{0}$, and consequently

$$
z F^{\prime}(z)+\alpha F(z) \ll \alpha \frac{1+z^{m}}{\left(1-z^{m}\right)^{1+2 \alpha / m}} .
$$

On comparing coefficients we have

$$
\begin{aligned}
\left|(m n+\alpha) A_{n}\right| & \leqq \alpha\left[\left(\begin{array}{c}
2 \alpha / m+n \\
n
\end{array}\right)+\left(\begin{array}{c}
2 \alpha / m+n-1 \\
n-1
\end{array}\right)\right] \\
\left|A_{n}\right| & \leqq\left(\begin{array}{c}
2 \alpha / m+n-1 \\
n
\end{array}\right),
\end{aligned}
$$

or equivalently, $[\phi(z) / z]^{\alpha} \ll\left(1-z^{m}\right)^{-2 \alpha / m}$. 
Corollary. If $\phi \in B_{m}(1 / N)$, where $N$ is a positive integer and $\phi(z)=z+a_{m+1} z^{m+1}+\cdots$ then

$$
\left|a_{m n+1}\right| \leqq\left(\begin{array}{c}
2 / m+n-1 \\
n
\end{array}\right) \text {. }
$$

In particular for $\phi \in B(1 / N)$ we have $\left|a_{n}\right| \leqq n$ and for $\phi \in B_{2}(1 / N)$ we have $\left|a_{2 n+1}\right| \leqq 1$.

Proof. With $\alpha=1 / N$ we have

$$
[\phi(z) / z\rfloor^{1 / N} \ll\left(1-z^{m}\right)^{-2 / m N} .
$$

Multiplying this result by itself $N$ times we obtain $\phi(z) / z$ $\ll\left(1-z^{m}\right)^{-2 / m}$, which is equivalent to the desired result.

The inequality (9) is sharp, as can be seen by considering the function $z\left(1-z^{m}\right)^{-2 / m}$ which is in $B_{m}(1 / N)$.

We conclude by constructing an example of a function $f \in B(1 / 2)$ such that $f$ is not close-to-convex. With an appropriate $c>0$, let $w=\phi(z)=z+\cdots$ be the odd close-to-convex function that maps $\Delta$ onto the $w$-plane slit along the half-lines $\operatorname{Re} w \geqq 0, \operatorname{Im} w=c$ and $\operatorname{Re} w \leqq 0, \operatorname{Im} w=-c$. Since $\phi \in B_{2}(1)$, by Theorem 1 we have $\phi(z)$ $=\left[f\left(z^{2}\right)\right]^{1 / 2}$, where $f(z) \in B(1 / 2)$. But the transformation $\zeta=\xi+i \eta$ $=f(z)$ maps $\Delta$ onto the $\zeta$-plane slit along the portion of the parabola $\xi=(\eta / 2 c)^{2}-c^{2}$ defined for $\eta \geqq 0$, and this slit clearly cannot be expressed as a union of half-lines. It follows by a well-known geometric criterion (see, for example, Bielecki and Lewandowski [2, p. 61]) that the domain is not close-to-convex.

\section{REFERENCES}

1. I. E. Bazilevič, On a case of integrability in quadratures of the Loewner-Kufarev equation, Mat. Sb. 37(79) (1955), 471-476. (Russian) MR 17, 356.

2. A. Bielecki and Z. Lewandowski, Sur un theorème concernant les fonctions univalentes linéairement asccessibles de M. Biernacki, Ann. Polon. Math. 12 (1962), 61-63. MR 26 \#5151.

3. G. Birkhoff and G. Rota, Ordinary differential equations, 2nd, ed., Blaisdell, Waltham, Mass., 1969. MR 38 \#4737.

4. Z. Nehari, Conformal mapping, McGraw-Hill, New York, 1952. MR 13, 640.

5. C. Pommerenke, On the coefficients of close-to-convex functions, Michigan Math. J. 9 (1962), 259-269. MR 26 \#5153.

6. - Über die Subordination analytischer Funktionen, J. Reine Angew. Math. 218 (1965), 159-173. MR 31 \#4900.

7. J. Zamorski, On Bazilevic schlicht functions, Ann. Polon. Math. 12 (1962), 8390. MR $26 \# 1446$.

UNIVERSTTY OF KeNTUCKy, LEXINGTon, KentuCKy 40506

State University of New York, Brockport, New York 14420 